\title{
Algorithm for Hybrid Optical Fiber-Wireless Photonic Channel Allocation for Millimeter-waveband 5G Networks
}

\section{Gomez Gonzalvo, A.; Vegas Olmos, Juan José; Tafur Monroy, Idelfonso}

\section{Published in:}

Proceedings of Asia Communications and Photonics Conference

Link to article, DOI:

10.1364/ACPC.2014.ATh3A.146

Publication date:

2014

Link back to DTU Orbit

Citation (APA):

Gomez Gonzalvo, A., Vegas Olmos, J. J., \& Tafur Monroy, I. (2014). Algorithm for Hybrid Optical Fiber-Wireless Photonic Channel Allocation for Millimeter-waveband 5G Networks. In Proceedings of Asia Communications and Photonics Conference Optical Society of America. https://doi.org/10.1364/ACPC.2014.ATh3A.146

\section{General rights}

Copyright and moral rights for the publications made accessible in the public portal are retained by the authors and/or other copyright owners and it is a condition of accessing publications that users recognise and abide by the legal requirements associated with these rights.

- Users may download and print one copy of any publication from the public portal for the purpose of private study or research.

- You may not further distribute the material or use it for any profit-making activity or commercial gain

- You may freely distribute the URL identifying the publication in the public portal 


\title{
Algorithm for Hybrid Optical Fiber-Wireless Photonic Channel Allocation for Millimeter-waveband 5G Networks
}

\author{
A. Gomez Gonzalvo, J. J. Vegas Olmos, and I. Tafur Monroy \\ Department of Photonics Engineering, Technical University of Denmark, Ørsted Plads 343, Kgs. Lyngby, 2800, Denmark \\ jjvo@fotonik.dtu.dk
}

\begin{abstract}
This paper presents a performance assessment of an algorithm for hybrid fiber-wireless photonic channel allocation in $5 \mathrm{G}$ using radio-over-fiber with active delivery. Simulations show reductions of network blocking probability in $98 \%$ of the tested cases.

OCIS codes: (060.2360) Fiber optics links and subsystems; (060.4251) Networks, assignment and routing algorithms.
\end{abstract}

\section{Introduction}

Radio-over-fiber (RoF) networks providing millimeter-waveband $(\mathrm{mmW})$ signals using wavelength-divisionmultiplexing (WDM) techniques are foreseen as an upcoming new paradigm to provide broadband wireless access in future 5G systems [1]. In such systems, due to the limited reach of $\mathrm{mmW}$ signals, it is expected that a single central station (CS) will need to support a large number of access points (APs). Generally, wireless networks present traffic-load profiles that are different than for fixed networks, mainly due to the mobility of the user. Hence, although signal distribution of WDM RoF signals is feasible, the challenge remains how to accommodate such a physical layer to the networking requirements of an access or $\mathrm{mmW}$ distribution network, particularly considering the traffic profiles [2]. Research on this topic had typically focused on the study of dynamic-bandwidth-allocation (DBA) protocols [3], which are implemented at the higher layers of the network-layer stack. However, in order to take full advantage of the bandwidth available in the optical domain, physical layers that can support reconfigurability seem to be a must for future RoF access in $5 \mathrm{G}$ networks. This paper presents an algorithm for a channel allocation technique operating over a hybrid distribution RoF network.

\section{Channel allocation techniques and proposed algorithm}

Different channel allocation techniques have been developed to manage the distribution of the channels among the different APs. There are three types of channel allocation strategies: Fixed Channel Allocation (FCA), Dynamic Channel Allocation (DCA) and Hybrid Channel Allocation (HCA).

In a FCA-based network, the distribution of the channels is permanent (or static), and established according to a previous frequency planning. On the other hand, DCA allows the allocation of the channels depending on the requirements of the different cells on-the-fly. All the channels are liable to be changed from one cell to another one, following an algorithm satisfying certain criteria, e.g. dropping prob. reduction [4] or handover occurrence reduction [5]. HCA merges the two above-mentioned techniques, operating with a set of fixed channels and a set of dynamic ones. The fixed channels are distributed among the different cells of the network following an FCA approach. The dynamic channels are kept in a pool shared by all the cells, allocating the different channels depending on the demands of the network. HCA therefore enables reconfigurability and flexibility while maintaining contained the technological complexity at the physical layer by avoiding full reconfigurability required in DCA approaches.

The proposed algorithm for Hybrid Channel Allocation of photonic channels in a $\mathrm{mmW}$ RoF environment is described next. The algorithm tackles the distribution of the channels in an access network, i.e. from the CS to the different Aps with the aim to reduce the overall blocking probability of the network. The blocking probability describes the fraction of time that a connection is denied due to insufficient available resources in the network. A FCA-based network is likely to have high blocking probabilities, due to a not-even distribution of the users among the different APs. The proposed algorithm for HCA will decide the allocation of the dynamic channels set in order to reduce the global blocking probability of the network. In the extension of Kendall's notation, each AP can be modeled as a M/M/N/N, assuming Poisson arrival rate, exponential service time and $\mathrm{N}$ servers which can handle at most $\mathrm{N}$ users. Equation (1) shows the Erlang-B formula, where $\lambda$ is the arrival rate, $\mu$ is the finishing rate and $N$ is the number of servers at the AP.

$$
p_{b}=\frac{\frac{1}{N !}\left(\frac{\lambda}{\mu}\right)^{N}}{\sum_{k=0}^{N} \frac{1}{k !}\left(\frac{\lambda}{\mu}\right)^{k}}
$$


The number of servers $\mathrm{N}$ directly depends on the number of channels allocated in an AP. Equation (1) also shows that as $\mathrm{N}$ increases, the blocking probability is reduced. Therefore, the allocation of channels will be done depending on the number of users at each AP at a certain time, allocating more dynamic channels into the most crowded APs.

The flowchart of the proposed algorithm is depicted in Fig. 1. The proposed method calculates the best distribution of dynamic channels in a time slot to reallocate the channels in the subsequent time slot. The optimum distribution of channels is the one in which the addition of the blocking probabilities of each AP of the network results in a minimum.

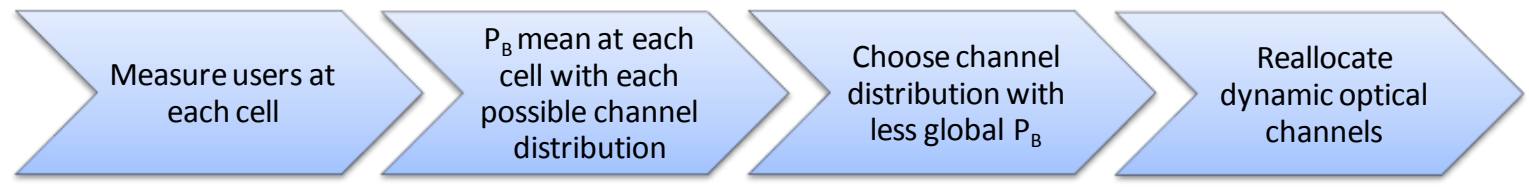

Fig. 1. Flowchart of the algorithm for reallocation of dynamic channels. $\mathrm{P}_{\mathrm{B}}$ : Blocking probability.

The algorithm requires the distribution of users among the different APs within a certain period of time. This period of time will define the frequency in which the dynamic channels are reallocated (Section 3). Once the distribution of the users is known, the blocking probability over time is computed using the Erlang-B Equation (1).

The next step is to compute the blocking probability with all the possible distributions of dynamic channels. To obtain a figure of merit of the evolution of $p_{b}$ over the time with each possible channel allocation, a weighted mean is applied for each channel distribution. The expression of the weighted mean is shown in Equation (2), where M refers to the size of the time slot.

$$
\overline{P_{B}}=\sum_{i=0}^{M} \frac{1}{2^{i}} p_{b}(M-i)
$$

The criterion to decide the next reallocation of channels is straightforward: the distribution which leads to the minimum blocking global probability mean of the network is chosen. The chosen distribution will be allocated in the subsequent time slot, where the algorithm will be run again.

\section{Performance evaluation}

For simulation purposes, an access network of two APs was taken. A random number of users were generated in each cell, limited to a maximum of 1000 users. The arrival rate was proportional to the number of users at each AP, and the finishing rate was $\mu=0.1$ calls/s. Each channel (fixed or dynamic) handled 8 servers. Each server was able to give service to one user at the same time. Fig. 2 shows the simulation results for the blocking probability over the time in two different scenarios, in a single test. The blocking probability is plotted over the time in the two different APs of the network. The reallocation time of the photonic channels was 200 seconds.
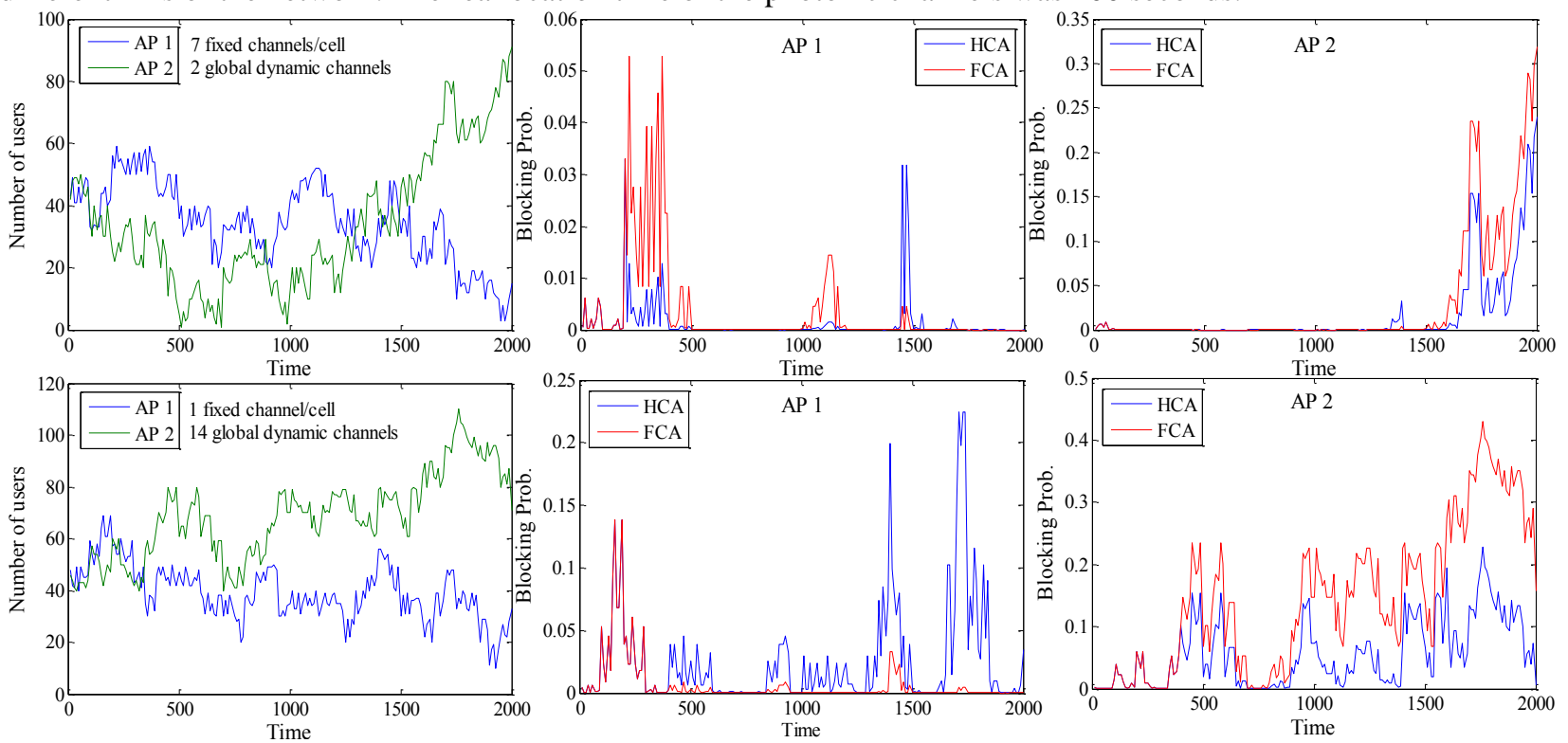

Fig. 2. Simulation results for two different scenarios, with (top) 7 fixed channels/cell and 2 global dynamic channels and (bottom) 1 fixed channel/cell and 14 global dynamic channels. AP: Access Point, FCA: Fixed Channel Allocation, HCA: Hybrid Channel Allocation 
The method was tested statistically, assessing the percentage of global blocking probability reduced by using in the same conditions the proposed algorithm in a HCA-network instead of using a FCA-based one. The reduction of the blocking probability was calculated as (3).

$$
\%_{\text {red }}=\left(1-\frac{\overline{P_{B}} H C A}{\overline{P_{B}} F C A}\right) \cdot 100
$$

Fig. 3 plots the histogram of the percentage of blocking probability reduction in two different scenarios. 1000 single tests with a different random number of users were done, assessing the performance of the algorithm in each of them. Each test simulated 2000 seconds of the network, with a channel reallocation every 200 seconds.

The first scenario had 7 fixed channels per cell and 2 dynamic channels to be distributed among both cells, whereas the second one had 1 fixed channel per cell and 14 global dynamic channels.

In the first scenario, 987 of the 1000 cases tested the algorithm for HCA improved the performance of a FCA network, ensuring a global blocking probability reduction. In an $83 \%$ of the times tested, the effective reduction of the blocking probability was less than $10 \%$.

In the second scenario, the blocking probability performance improved in relation to the first one. The values of blocking probability reduction are distributed more uniformly. Whereas in the first scenario the effective reduction between $40 \%$ and 50\% was 7 times, in the second one it was 34 times. In this scenario, the HCA network based on our algorithm was 28 times worse than the FCA one, experiencing an increase of the global blocking probability.
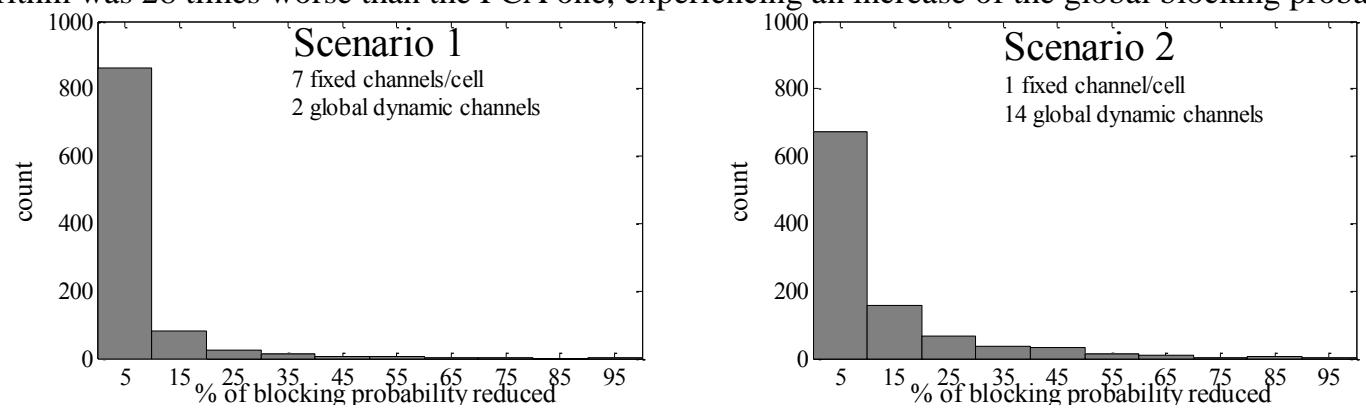

Fig. 3. Histogram of blocking probability reduction by using HCA instead of FCA, with (left) 7 fixed channels per cell and 2 global dynamic channels and (right) 1 fixed channel per cell and 14 global dynamic channels

\section{Conclusion}

We developed a novel algorithm for the dynamic allocation of the photonic channels in a RoF network. The algorithm was assessed by computer simulation, ensuring a blocking probability reduction of the network in a $98 \%$ of the cases tested. The man value of the blocking probability reduction in the first scenario was $5.19 \%$, whereas in the second one was $11.25 \%$, so a better performance was achieved with a higher ratio of dynamic versus fixed channels. However, the technological complexity at the physical layer also increases. We have proposed a novel future implementation for the allocation of the photonic channels which would ensure a blocking probability reduction in next generation access $5 \mathrm{G}$ wireless networks.

\section{Acknowledgements}

The authors thank the European Commission for partly funding this research through the Marie Curie FENDOI project and the FP7 IPHOBAC-NG project.

\section{References}

[1] F. Boccardi, R. W. Heath, A. Lozano, T. L. Marzetta, and P. Popovski, "Five Disruptive Technology Directions for 5G," IEEE Communications Magazine, Special Issue on "5G Wireless Communication Systems: Prospects and Challenges" 52 (2), 74-80 (2014).

[2] G. Kalfas, and N. Pleros, "An agile and medium-transparent MAC protocol for $60 \mathrm{GHz}$ radio-over-fiber local access networks," IEEE Journal of Lightwave Technology 28(16). 2315-2326 (2010).

[3] M. P. McGarry, M. Maier, and M. Reisslein, "Ethernet PONs: A survey of dynamic bandwidth allocation (DBA) algorithms," IEEE Communications Magazine 42, S8-S15 (2004).

[4] T. Zhang, and P. Agarwal, "Local predictive resource reservation for handoff in multimedia wireless networks," IEEE Journal on Selected Areas in Communications 19(10), 1931-1941 (2001).

[5] L. Ortigoza-Guerrero, and A. H. Aghvami, "A Prioritized Handoff Dynamic Channel Allocation Strategy for PCS," IEEE Transactions on Vehicular Technology 48(4), 1203-1215 (1999). 